\title{
NONLINEAR MULTIVARIABLE CONTROL OF AN OMNIDIRECTIONAL VEHICLE
}

\author{
Frédérick Bourgoin ${ }^{1}$ André Desbiens ${ }^{1}$
}

\author{
LOOP (Laboratoire d'observation et d'optimization des \\ procédés - Process observation and optimization laboratory) \\ Department of Electrical and Computer Engineering \\ Université Laval, Pavillon Adrien-Pouliot \\ Québec (Québec), Canada G1K $7 P_{4}$ \\ Email: desbiens@gel.ulaval.ca
}

\begin{abstract}
This paper describes the multi-level control of a three-wheel omnidirectional vehicle. The vehicle was developed to be an autonomous robotic soccer player with respect to the Robocup F180 small-size league rules. The two inner control levels are model nonlinear reference controllers (MNRC) while the last level of control relies on a modified Smith Predictor. The paper covers the modeling of the vehicle, the parameter identification using an output-error approach, and the design and implementation of the controllers. Some results illustrate the good performances. Copyright (C) 2005 IFAC
\end{abstract}

Keywords: Vehicle, robot control, nonlinear control, multivariable control.

\section{INTRODUCTION}

A three-wheel omnidirectional vehicle offers the advantage to fully cover the three degrees of freedom of a planar movement $(x-y$ position and angle). However, for adequate control performances, multivariable nonlinear techniques are required. The objective is to control the $x-y$ position and the angle of the vehicle relatively to a fixed referential.

The proposed approach is a three-level hierarchic control structure. The inner multivariable nonlinear loop controls the angular speed of the three wheels. The second multivariable nonlinear controller regulates the speed of the vehicle in a fixed referential. Finally, the last level of control allows the tracking of the position and angle in a fixed referential with a modified Smith Predictor controller.

\footnotetext{
1 Partially supported by NSERC (Canada).
}

The next section details the physical model of the vehicle. It relies on kinetic and electromechanical equations. Section 3 describes the parameter identification procedure to adjust some parameters of the preceding model. Section 4 details the control structure.

\section{THE OMNIDIRECTIONAL VEHICLE}

\subsection{Physical description}

Each vehicle wheel produces a standard traction perpendicular to the rotation axis while presenting virtually no friction in the direction parallel to the axis (Figure 1). A combination of three of these wheels insures a complete coverage of the three degrees of freedom in a plan. Each wheel is independently driven by a DC motor. The power is transmitted to each motor using a H-bridge modulated by a PWM generator, which duty cycle varies according to the desired output power. The 

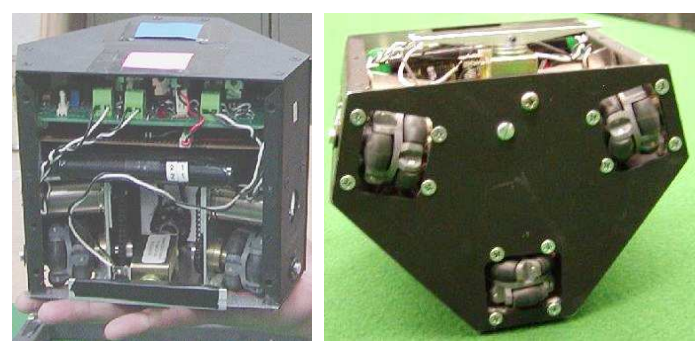

Fig. 1. Front and under view of the omnidirectionnal vehicle

wheel angular positions are measured with rotary encoders. A processing unit allows an on-board implementation of the first level of control (wheel speeds).

A camera is located 3 meters over a green field on which the vehicle moves. A color pattern on the top of the vehicle allows the camera to determine the vehicle position and orientation. An external computer processes the images, computes the second and third levels of control and sends the control variables (wheel speed set points) to the vehicle through unidirectional wireless communications.

\subsection{Model}

Vehicle accelerations are produced by the vectorial sum of wheel tractions. To develop the model, the following equations are required:

(1) The relationship between the wheel velocity vector and the vehicle mass center speed;

(2) The expression of the force generated by each wheel as a function of the motor input voltage;

(3) The vehicle mass center acceleration according to the force produced by each wheel.

Figure 2 shows the main variables of the vehicle model as well as the vehicle $(V)$ and fixed $(F)$ referentials. The referential $V^{*}$ is the vehicle referential reported to the origin of the fixed referential in order to show the definition of $\varphi_{c}^{F}$. For omnidirectional mobile vehicles, an instantaneously coincident coordinate system should be used to specify the velocity of the vehicle independently of its position (Muir, 1990). Then, an instantaneously coincident coordinate system $\bar{V}$ is defined to be at the same position and orientation in space as the $V$ coordinate system, but stationary with respect to the fixed $F$ coordinate system. The transformation matrix used to transform a coordinate from the fixed referential to the $\bar{V}$ coordinate system is:

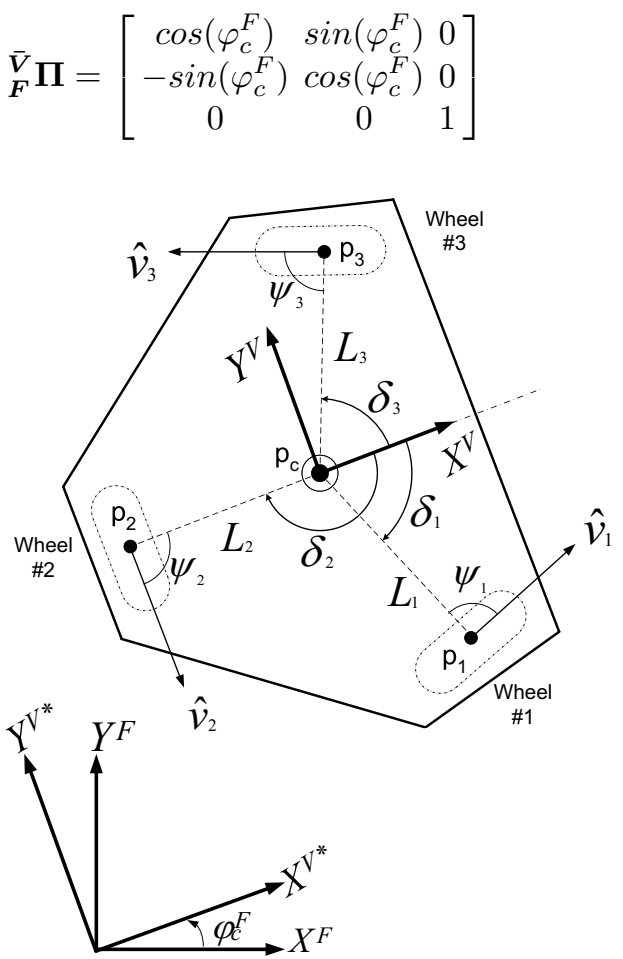

Fig. 2. Referentiel definition

Kinetic equations are used to link the wheel angular speed, $\boldsymbol{\omega}=\left[\begin{array}{lll}\omega_{1} & \omega_{2} & \omega_{3}\end{array}\right]^{T}$, to the robot mass center speed in the fixed frame, $\dot{\boldsymbol{p}}_{\boldsymbol{c}}^{\boldsymbol{F}}=\left[\begin{array}{lll}\dot{x}_{c}^{F} & \dot{y}_{c}^{F} & \dot{\varphi}_{c}^{F}\end{array}\right]^{T}$ $\left(x_{c}^{F}\right.$ and $y_{c}^{F}$ are the positions and $\varphi_{c}^{F}$ is the angle, all in the fixed referential). The speed of wheel $i$, $\omega_{i}$, is function of the speed of its point of contact with the ground, $\dot{\boldsymbol{p}}_{\boldsymbol{i}}^{\boldsymbol{F}}$, as:

$$
\omega_{i}=\frac{\dot{\boldsymbol{p}}_{\boldsymbol{i}}^{\boldsymbol{F}} \cdot \hat{\boldsymbol{v}}_{\boldsymbol{i}}}{r}
$$

where $r$ is the wheel radius and $\hat{\boldsymbol{v}}_{\boldsymbol{i}}$ is the traction direction vector, which is perpendicular to the wheel axis. From Figure 2, one can deduce:

$$
\hat{\boldsymbol{v}}_{\boldsymbol{i}}=\left[\begin{array}{l}
-\cos \left(\varphi_{c}^{F}+\delta_{i}-\psi_{i}\right) \\
-\sin \left(\varphi_{c}^{F}+\delta_{i}-\psi_{i}\right)
\end{array}\right]
$$

and

$$
\boldsymbol{p}_{\boldsymbol{i}}^{\boldsymbol{F}}=\left[\begin{array}{l}
x_{c}^{F}+L_{i} \cos \left(\varphi_{c}^{F}+\delta_{i}\right) \\
y_{c}^{F}+L_{i} \sin \left(\varphi_{c}^{F}+\delta_{i}\right)
\end{array}\right]
$$

and thus

$$
\dot{\boldsymbol{p}}_{\boldsymbol{i}}^{\boldsymbol{F}}=\left[\begin{array}{c}
\dot{x}_{c}^{F}-\dot{\varphi}_{c}^{F} L_{i} \sin \left(\varphi_{c}^{F}+\delta_{i}\right) \\
\dot{y}_{c}^{F}+\dot{\varphi}_{c}^{F} L_{i} \cos \left(\varphi_{c}^{F}+\delta_{i}\right)
\end{array}\right]
$$

From equations (2), (3) and (5), the wheel angular speed is obtained:

$$
\boldsymbol{\omega}=\frac{1}{r} \cdot \boldsymbol{M}_{\boldsymbol{C}} \cdot \overline{\boldsymbol{V}} \boldsymbol{\Pi} \cdot \dot{\boldsymbol{p}}_{\boldsymbol{c}}^{\boldsymbol{F}}
$$

where:

$$
\boldsymbol{M}_{\boldsymbol{C}}=\left[\begin{array}{lll}
-\cos \left(\alpha_{1}\right) & -\sin \left(\alpha_{1}\right) & L_{1} \sin \left(\psi_{1}\right) \\
-\cos \left(\alpha_{2}\right) & -\sin \left(\alpha_{2}\right) & L_{2} \sin \left(\psi_{2}\right) \\
-\cos \left(\alpha_{3}\right) & -\sin \left(\alpha_{3}\right) & L_{3} \sin \left(\psi_{3}\right)
\end{array}\right]
$$

with

$$
\alpha_{i}=\delta_{i}-\psi_{i}
$$


Electromechanical equations will lead to the relationship between forces produced by each wheel on the ground, $\boldsymbol{f}=\left[\begin{array}{lll}f_{1} & f_{2} & f_{3}\end{array}\right]^{T}$, the input voltage, $\boldsymbol{v}=\left[\begin{array}{lll}v_{1} & v_{2} & v_{3}\end{array}\right]^{T}$ and the wheel velocity, $\boldsymbol{\omega}$. Forces produced by each wheel on the ground are:

$$
f_{i}=\frac{t_{i}}{r}
$$

where $t_{i}$ is the resultant torque of wheel $i$. Torque $t_{m i}$ produced by a small DC motor when the inductance is neglected, is given by (Nise S., 2000):

$$
t_{m i}=\frac{k_{t} \cdot v_{i}}{r_{a}}-\frac{k_{t} \cdot k_{b} \cdot \omega_{m i}}{r_{a}}
$$

where $k_{t}$ is the torque coefficient of the DC motor, $k_{b}$ is the back-emf coefficient of the DC motor, $r_{a}$ is the electrical resistance of the DC motor and $\omega_{m i}$ is the angular speed of the motor $i$. Considering a gearbox ratio $n_{g b}$ between each motor and wheel, using equation (9) and (10) and neglecting internal inertia and damping of the motor-wheel system, because they will be overshadowed by the vehicle inertia, the forces are:

$$
f=M_{A} \cdot v-M_{B} \cdot \omega
$$

where:

$$
\begin{aligned}
& \boldsymbol{M}_{\boldsymbol{A}}=\boldsymbol{I}_{\mathbf{3} \boldsymbol{x} \mathbf{3}} \cdot \frac{1}{r} \cdot\left(\frac{k_{t} \cdot n_{g b}}{r_{a}}\right) \\
& \boldsymbol{M}_{\boldsymbol{B}}=\boldsymbol{I}_{\mathbf{3} \boldsymbol{x} \mathbf{3}} \cdot \frac{1}{r} \cdot\left(\frac{k_{t} \cdot K_{b} \cdot n_{g b}^{2}}{r_{a}}\right)
\end{aligned}
$$

Dynamic equations, linking the vehicle mass center acceleration, $\ddot{\boldsymbol{p}}_{\boldsymbol{c}}^{\boldsymbol{F}}$, to the forces $\boldsymbol{f}$ applied at $p_{1}$, $p_{2}$ and $p_{3}$ are obtained with Newton's law. The vehicle angular acceleration is:

$$
\ddot{\varphi}_{c}^{F}=\sum_{i=1}^{3} t_{v i} / j_{v}
$$

where $t_{v i}$ is the resultant torque produced by the wheel on the vehicle mass center and $j_{v}$ is the vehicle rotational inertia. From Figure (2):

$$
t_{v i}=L_{i} f_{i} \sin \left(\psi_{i}\right)
$$

The vehicle linear accelerations are:

$$
\begin{aligned}
& \ddot{x}_{c}^{F}=\sum_{i=1}^{3} f_{i x}^{F} / m \\
& \ddot{y}_{c}^{F}=\sum_{i=1}^{3} f_{i y}^{F} / m
\end{aligned}
$$

where $f_{i x}^{F}$ and $f_{i y}^{F}$ are the forces produced by wheel $i$ on the vehicle mass center in the $x$ and $y$ directions respectively, in the fixed referential, and $m$ is the vehicle mass. From Figure (2), one obtains:

$$
f_{i x}^{F}=-f_{i} \cos \left(\varphi_{c}^{F}+\delta_{i}-\psi_{i}\right)
$$

$$
f_{i y}^{F}=-f_{i} \sin \left(\varphi_{c}^{F}+\delta_{i}-\psi_{i}\right)
$$

These last six equations result in:

$$
\ddot{p}_{c}^{F}=M_{M} \cdot{ }_{F}^{\bar{V}} \Pi^{T} \cdot M_{\boldsymbol{C}}^{\boldsymbol{T}} \cdot \boldsymbol{f}
$$

where:

$$
\boldsymbol{M}_{\boldsymbol{M}}=\left[\begin{array}{ccc}
1 / m & 0 & 0 \\
0 & 1 / m & 0 \\
0 & 0 & 1 / J_{v}
\end{array}\right]
$$

To develop the wheel angular speed controller, $\boldsymbol{p}_{\boldsymbol{c}}^{\boldsymbol{F}}$ must be defined in the vehicle coordinate system, in order to become independent of $\varphi_{c}^{F}$. Applying

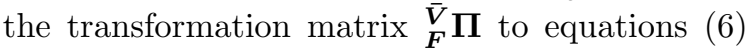
and (20) and combining them with equation (11) lead to:

$$
\dot{\omega}=M_{1} \cdot v-M_{2} \cdot \omega
$$

where:

$$
M_{1}=\frac{1}{r} M_{C} M_{M} M_{C}^{T} M_{A}
$$

and

$$
M_{2}=\frac{1}{r} M_{C} M_{M} M_{C}^{T} M_{B}
$$

Note that equation (22) is independent of $\varphi_{c}^{F}$.

\section{IDENTIFICATION}

To adequately design a model-based controller, a dynamic model that can precisely predict the controlled variables for manipulated variable moves is required. The process to be identified is excited by manipulating the inputs (manipulated variables) and recording the outputs (controlled variables). According to an output-error approach, the model parameters are identified by minimizing (Ljung, 1999):

$$
J=\sum_{k=1}^{N}\left[\boldsymbol{y}_{\boldsymbol{m}}(k)-\boldsymbol{y}_{\boldsymbol{p}}(k)\right]^{T}\left[\boldsymbol{y}_{\boldsymbol{m}}(k)-\boldsymbol{y}_{\boldsymbol{p}}(k)\right]
$$

where $N$ is the number of recorded samples, $\boldsymbol{y}_{\boldsymbol{p}}(k)$ is the recorded process output vector and $\boldsymbol{y}_{\boldsymbol{m}}(k)$ is the predicted model output vector for the given process inputs. For better results, the inputs should be rich at the frequencies where precision is necessary. Various input amplitudes are also required in presence of nonlinearities. The resulting model should be validated with data that were not used for identification.

\section{MULTIVARIABLE NONLINEAR CONTROL}

\subsection{Control structure}

Figure 3 shows the complete hierarchical control structure. The wheel speed vector $\boldsymbol{\omega}$ is controlled 
by manipulating the voltage vector $\boldsymbol{v}$ with the inner controller, physically located onboard. The vehicle mass center speed in the fixed frame $\dot{\boldsymbol{p}}_{\boldsymbol{c}}^{\boldsymbol{F}}$ is regulated by manipulating the wheel speed set points $\boldsymbol{r}_{\boldsymbol{\omega}}$ with a second controller (Figure 4). The mass center speed is measured with the camera and processing the images generates a dead time. Therefore, a Smith predictor (Smith, 1957) is used to handle this delay. The second controller is implemented in a computer to which the camera is connected. The wheel set points $\boldsymbol{r}_{\boldsymbol{\omega}}$ is sent to the vehicle through RF communications. Finally, the mass center position $\boldsymbol{p}_{c}^{F}$ is controlled by a third controller that also includes a Smith predictor (Figure 5) which is modified to handle the presence of an integrator in the process (Hang et al., 2003). This control is also implemented in the computer. The manipulated variables are the set points of the second controller, i.e. the vehicle speed set points $\boldsymbol{r}_{\boldsymbol{v}}$. The vehicle position set point vector is denoted $\boldsymbol{r}_{\boldsymbol{p}}$.

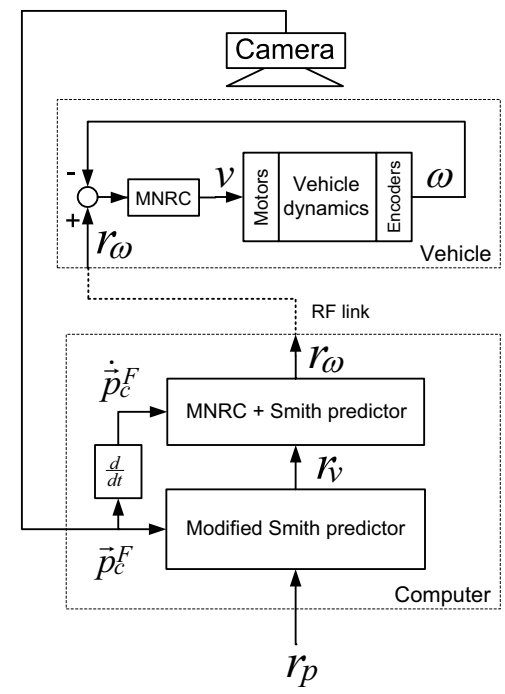

Fig. 3. Control structure

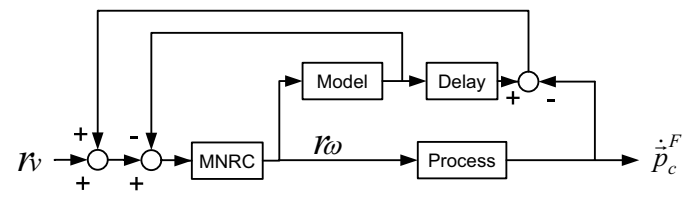

Fig. 4. Second level controller

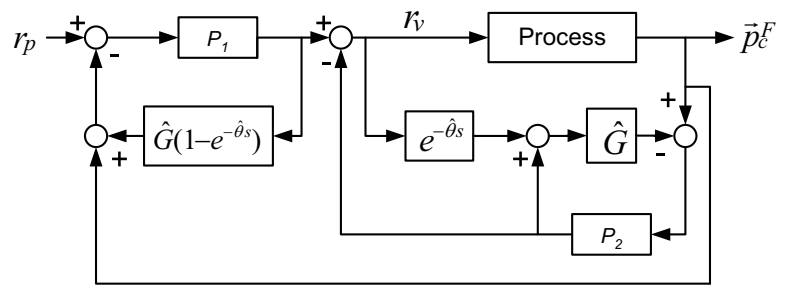

Fig. 5. Modified Smith Predictor

The first two levels of control are model nonlinear reference controllers (MNRC) with integral action (Chidambaram, 1995). MNRC basically inverses the process model leading to decoupled tracking performances selected by reference models. The model of the process to be controlled is supposed to be the following:

$$
\begin{aligned}
& \dot{\boldsymbol{x}}=f(\boldsymbol{x})+g(\boldsymbol{x}) \cdot \boldsymbol{u} \\
& \boldsymbol{y}=h(\boldsymbol{x})
\end{aligned}
$$

The MNRC control law with integral action is:

$$
\boldsymbol{u}=(\boldsymbol{J} g(\boldsymbol{x}))^{-1}\left(\dot{\boldsymbol{y}}_{\boldsymbol{m}}-\boldsymbol{J} f(\boldsymbol{x})+\boldsymbol{k} \boldsymbol{e}+\boldsymbol{k}_{\boldsymbol{I}} \boldsymbol{e}_{\boldsymbol{I}}\right)
$$

where $\boldsymbol{J}=d h(\boldsymbol{x}) / d \boldsymbol{x}$ and the tracking reference trajectory $\boldsymbol{y}_{\boldsymbol{m}}$ is defined by:

$$
\dot{y}_{m}=\Lambda_{m} y_{m}-\Lambda_{m} y_{r}
$$

where $\boldsymbol{y}_{\boldsymbol{r}}$ is the set point and $\boldsymbol{\Lambda}_{\boldsymbol{m}}$ is the eigenvalue diagonal matrix fixing the tracking reference trajectory dynamics. The error vector is $\boldsymbol{e}=\boldsymbol{y}_{\boldsymbol{m}} \boldsymbol{- \boldsymbol { y }}$ and its integration is $\boldsymbol{e}_{\boldsymbol{I}}=\int \boldsymbol{e} d t$. The proportional and integral tuning parameters are $\boldsymbol{k}=\operatorname{diag}\left[\kappa_{i}\right]$ and $\boldsymbol{k}_{\boldsymbol{I}}=\operatorname{diag}\left[\kappa_{i} / \tau_{I i}\right]$. Tuning is calculated according to:

$$
\begin{aligned}
\kappa_{i} & =\frac{2 \zeta_{i}}{\tau_{i}} \\
\tau_{I i} & =2 \zeta \tau_{i}
\end{aligned}
$$

where $\zeta_{i}$ is the error damping coefficient and $\tau_{i}$ the error effective time constant which are both fixed according to the desired vanishing error dynamics.

\subsection{First level of control}

The MNRC controller is based on the model described by (22), the inputs being the voltage applied to the motors $\boldsymbol{v}$ and the outputs are the wheel speeds $\boldsymbol{\omega}$. The model must be adequately calibrated by the identification procedure described in Section 3. The identified parameters are the matrices $\boldsymbol{M}_{\mathbf{1}}$ and $\boldsymbol{M}_{\mathbf{2}}$. Figure 6 shows typical validation results with a $15 \mathrm{~ms}$ sampling period. For the model (22) to have the same structure as (26), the following must hold: $f(x)=-\boldsymbol{M}_{\mathbf{2}} \cdot \boldsymbol{\omega}$, $g(x)=\boldsymbol{M}_{\mathbf{1}}$ and $h(x)=\boldsymbol{\omega}$. Since $\boldsymbol{J}=\boldsymbol{I}_{\mathbf{3 x} \mathbf{3}}$ and with the tuning $\boldsymbol{\Lambda}_{\boldsymbol{\omega}}=\operatorname{diag}\left[\lambda_{\omega i}\right]_{i=1,2,3}$, the control law is then:

$$
v=M_{1}^{-1} \cdot\left(\dot{\omega}_{m}+M_{2} \cdot \omega+k_{w} \cdot e_{\omega}+k_{I \omega} \cdot e_{I \omega}\right)
$$

where:

$$
\dot{\omega}_{m}=\Lambda_{\omega} \cdot \omega_{m}-\Lambda_{\omega} \cdot r_{\omega}
$$

That controller leads to performances close to theoretical results:

$$
\boldsymbol{\omega}(s)=\operatorname{diag}\left[\frac{1}{1-s / \lambda_{w i}}\right]_{i=1,2,3} \boldsymbol{r}_{\boldsymbol{w}}(s)
$$

Figure 7 depicts the controller performances. As expected, almost perfect decoupling is achieved. 

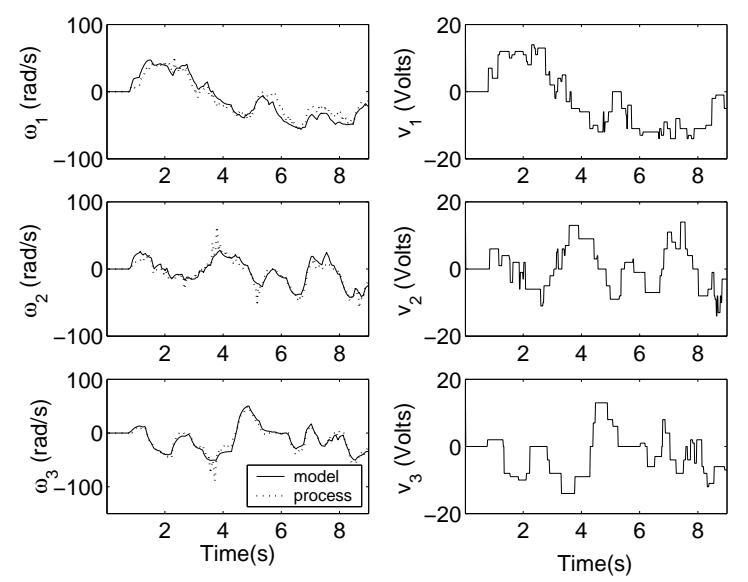

Fig. 6. Wheel speed model validation
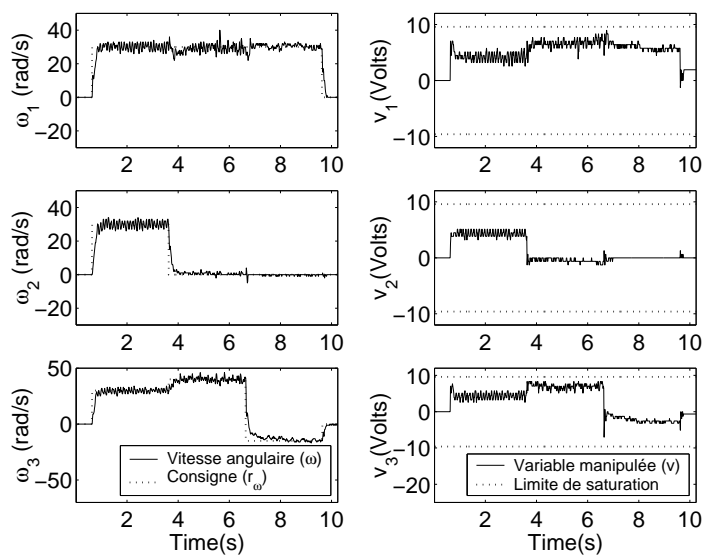

Fig. 7. Wheel speed controller validation

\subsection{Second level of control}

Considering (6) and the previous closed-loop dynamics (33), the model for the second MNRC is (it is assumed that all $\lambda_{w i}$ were selected identical):

$$
\ddot{p}_{c}^{F}=M_{3} \cdot \dot{p}_{c}^{F}-M_{3} \cdot{ }_{F}^{\bar{V}} \Pi^{-1} \cdot M_{4} \cdot r_{w}
$$

where $\boldsymbol{M}_{\mathbf{3}}=\operatorname{diag}\left[\lambda_{\omega}\right]$ and $\boldsymbol{M}_{\mathbf{4}}=r \cdot \boldsymbol{M}_{\boldsymbol{C}}^{-1}$. Since ${ }_{\boldsymbol{F}} \boldsymbol{\Pi}^{-1}$ is function of $\varphi_{c}^{F}$, the system is nonlinear and coupled. Parameter identification is performed to adjust $\boldsymbol{M}_{\mathbf{3}}$ and $\boldsymbol{M}_{\mathbf{4}}$.

Again, the above model is appropriate for the MNRC approach by setting $f(x)=\boldsymbol{M}_{\mathbf{3}} \cdot \dot{\boldsymbol{p}}_{\boldsymbol{c}}^{\boldsymbol{F}}$, $g(x)=-\boldsymbol{M}_{\mathbf{3}} \cdot{ }_{\boldsymbol{F}}^{\overline{\boldsymbol{F}}} \boldsymbol{\Pi}^{-1} \cdot \boldsymbol{M}_{\mathbf{4}}, h(x)=\dot{\boldsymbol{p}}_{\boldsymbol{c}}^{\boldsymbol{F}}$. Since $\boldsymbol{J}=\boldsymbol{I}_{\mathbf{3} \boldsymbol{x} \mathbf{3}}$ and $\boldsymbol{\Lambda}_{\boldsymbol{v}}=\operatorname{diag}\left[\lambda_{v i}\right]_{i=x, y, \varphi}$, the control law is:

$$
\boldsymbol{r}_{\boldsymbol{w}}=\left(-\boldsymbol{M}_{\mathbf{3}} \cdot \overline{\boldsymbol{V}} \boldsymbol{\Pi}^{-1} \cdot \boldsymbol{M}_{\mathbf{4}}\right)^{-1}\left(\begin{array}{l}
\ddot{p}_{\boldsymbol{m}}^{\boldsymbol{F}}-\boldsymbol{M}_{\mathbf{3}} \dot{p}_{\boldsymbol{c}}^{\boldsymbol{F}}+ \\
\boldsymbol{k}_{\boldsymbol{v}} \boldsymbol{e}_{\boldsymbol{v}}+\boldsymbol{k}_{\boldsymbol{I} \boldsymbol{v}} \boldsymbol{e}_{\boldsymbol{I} \boldsymbol{v}}
\end{array}\right)
$$

where:

$$
\ddot{p}_{m}^{F}=\Lambda_{v} \cdot \dot{p}_{m}^{F}-\Lambda_{v} \cdot r_{v}
$$

In order to deal with the delay $\theta$ caused by the vision system, a Smith predictor is added to the MNRC controller (Figure 4). The resulting theoretical closed-loop dynamics are then:

$$
\dot{\boldsymbol{p}}_{\boldsymbol{c}}^{\boldsymbol{F}}(s)=\operatorname{diag}\left[\frac{e^{-\theta s}}{1-s / \lambda_{v i}}\right]_{i=x, y, \varphi} \boldsymbol{r}_{\boldsymbol{v}}(s)
$$

where $\lambda_{v i}$ define the MNRC tracking reference model dynamics. Model validation is illustrated in Figure 8 and the performances of the controller are shown in Figure 9.
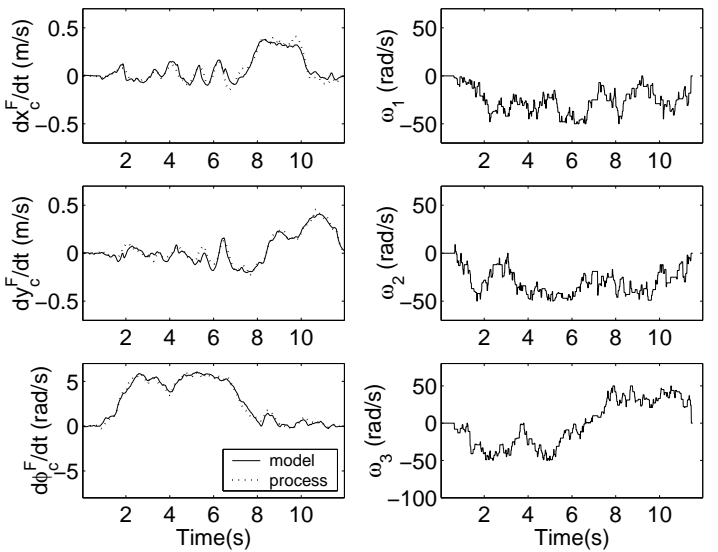

Fig. 8. Vehicle speed model validation
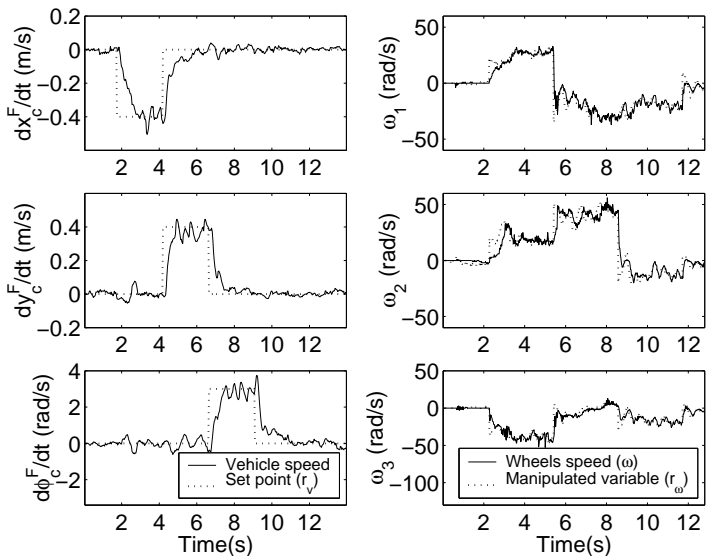

Fig. 9. Vehicle speed controller validation

\subsection{Third level of control}

The last level controls the vehicle position. If only speed needs to be controlled, the third level of control is not required. Integrating (37) leads to the model required for the design of the controller:

$$
\boldsymbol{p}_{c}^{F}(s)=\operatorname{diag}\left[\frac{e^{-\theta s}}{s\left(1-s / \lambda_{v i}\right)}\right]_{i=x, y, \varphi} \boldsymbol{r}_{\boldsymbol{v}}(s)
$$

The parameters $\lambda_{v i}$ are adjusted with the identification procedure to fit experimental data. The process being diagonal and linear, the control is also selected with these characteristics. To deal with the presence of an integrator and a long delay, a modified Smith predictor (Hang et al., 2003) is used (Figure 5). $P_{1}$ and $P_{2}$ are scalar selected to obtain desired second-order closed-loop dynamics. Figure 10 depicts typical control results. The vehicle performs a circle of $0.3 \mathrm{~m}$ radius in the fixed 
coordinate system while turning on itself. Those movements are only possible with an omnidirectionnal vehicle.
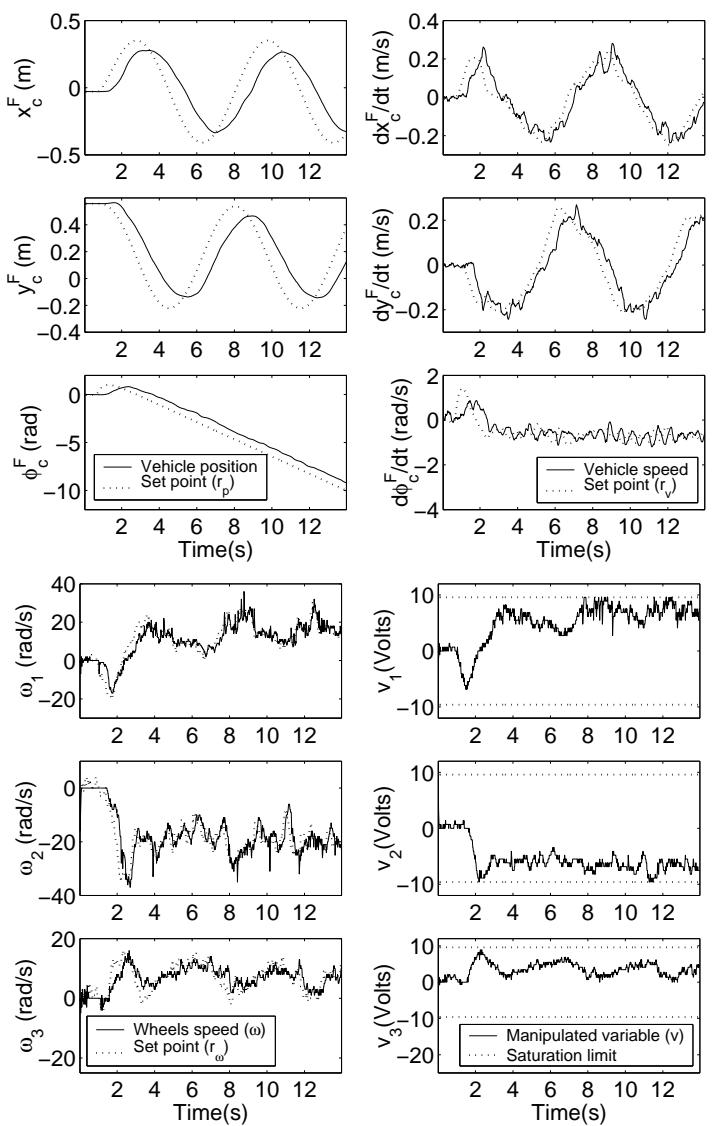

Fig. 10. Third level of control

\section{CONCLUSION}

A multilevel MNRC-based controller was used to control an omnidirectional nonlinear vehicle with very good performances. The MNRC control algorithm provides a simple and efficient approach for the control of multivariable nonlinear systems. Because of its integral action, model mismatches do not cause any harm in steady-state. Since MNRC inverses the process model, the resulting dynamics are decoupled hence making easier the design of the last level of control. Smith predictors are added to handle the delay caused by the vision system.

In order to reduce slipping and to be independent from an external camera, internal accelerometers or gyroscopes could be used to provide speed measurements. This would make the vehicle fully autonomous. Such a vehicle could then be easily modified for human needs such as wheelchairs or industrial lifts.

\section{REFERENCES}

Chidambaram, M. (1995). Nonlinear process control. John Wiley \& Sons. New Delhi.

Hang, C. C., Qing-Guo Wang and Xue-Ping Yang (2003). A modified smith predictor for a process with an integrator and long dead time. Industrial and Engineering Chemistry Research 42, pp. 484-489.

Ljung, Lennart (1999). System identification. Prentice Hall PTR. New Jersey.

Muir, Patrick (1990). Kinematic modeling for feedback control of an omnidirectional wheeled mobile robot. In: Autonomous Robot Vehicles (I.J. Cox and G.T. Wilfong, Eds.). pp. 25-31. Springer-Verlag. New York.

Nise S., Norman (2000). Control systems engineering: Third edition. John Wiley \& Sons. New York.

Smith, O.J.M (1957). Close control of loops with dead time. Chemical Engineering Progresses 53(5), pp. 217-219. 